\title{
A non-relativistic logarithmic conformal field theory from a holographic point of view
}

\section{Eric A. Bergshoeff, Sjoerd de Haan, Wout Merbis and Jan Rosseel}

Centre for Theoretical Physics, University of Groningen,

Nijenborgh 4, 9747 AG Groningen, The Netherlands

E-mail: E.A.Bergshoeff@rug.nl, s.de.haan@rug.nl, w.merbis@rug.nl,

j.rosseel@rug.nl

ABSTRACT: We study a fourth-order derivative scalar field configuration in a fixed Lifshitz background. Using an auxiliary field we rewrite the equations of motion as two coupled second order equations. We specialize to the limit that the mass of the scalar field degenerates with that of the auxiliary field and show that logarithmic modes appear. Using non-relativistic holographic methods we calculate the two-point correlation functions of the boundary operators in this limit and find evidence for a non-relativistic logarithmic conformal field theory at the boundary.

KEYWORDS: Gauge-gravity correspondence, Holography and condensed matter physics (AdS/CMT), AdS-CFT Correspondence

ARXiV EPRINT: 1106.6277 


\section{Contents}

1 Introduction 1

2 The model 3

2.1 General features 3

$\begin{array}{ll}2.2 & \text { An example: } z=2\end{array}$

3 Two point correlation functions $\quad 8$

3.1 Holographic renormalization 8

$\begin{array}{ll}3.2 & \text { Two-point correlation functions } \\ & 11\end{array}$

$\begin{array}{lll}3.2 .1 & \text { Example 1: } z=1 & 11\end{array}$

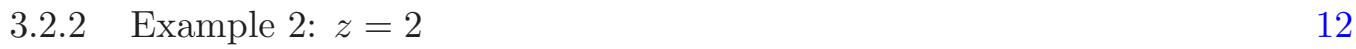

$\begin{array}{lll}3.3 & \text { Comparison with LCFT's } & 13\end{array}$

4 Conclusions 14

\section{Introduction}

Gauge/gravity dualities have taught us a lot about the properties of strongly coupled field theories. The most studied gauge/gravity duality is the AdS/CFT correspondence which deals with the gravitational description of conformal field theories [1]. Over the years generalizations of this correspondence have been proposed that are more closely connected to 'real life' physical systems, like the quark-gluon plasma or, more recently, condensed matter systems.

In the case of condensed matter physics, one conjectures a gravitational dual for field theories that exhibit an anisotropic scale invariance. Such an anisotropic scaling behaviour can be embedded in various symmetry algebras, such as the Lifshitz algebra [2], which consists of spatial rotations and translations, time translations and a scaling transformation, and the Schrödinger algebra [3, 4] which extends the former algebra with Galilean boosts and a number operator. Both algebras are characterized by a dynamical exponent $z$, which specifies how the scale transformations act differently on the time and spatial coordinates. Since algebras of this kind describe symmetries of non-relativistic field theories exhibiting non-relativistic dispersion relations, the corresponding gauge/gravity dualities are often stated as giving examples of non-relativistic holography.

An independent new development in gauge/gravity duality is the connection between logarithmic conformal field theories (LCFT) and critical gravity theories [5-16]. This connection was first made in the context of three-dimensional massive gravity theories, like Topologically Massive Gravity [17] or New Massive Gravity [18]. These are higherderivative three-dimensional gravity theories, where the Einstein-Hilbert action is supplemented with a negative cosmological constant and specific interactions with up to four 
derivatives. The spectrum of linearized perturbations of these theories is described by (unitary or non-unitary) massive bulk gravitons and boundary gravitons that do not describe any physical bulk degrees of freedom. At certain points in the parameter space of these theories, a degeneracy takes place and the massive gravitons coincide with the boundary gravitons. Such a special point is dubbed a "critical point" and the theory at such a critical point is referred to as a "critical gravity" theory. At the critical point, the massive gravitons are replaced by so called logarithmic modes. According to the AdS/CFT dictionary, the boundary gravitons are dual to the components of the stress-energy tensor of the boundary field theory. The logarithmic modes on the other hand source so-called logarithmic operators that degenerate with the components of the stress energy tensor in all quantum numbers. This results in a logarithmic conformal field theory, introduced in physics by [19]. ${ }^{1}$ A defining feature of a LCFT is that the Hamiltonian is no longer diagonalisable: the components of the stress energy tensor form a pair with the logarithmic operators and the action of the Hamiltonian on such a pair is not diagonalisable.

Although the connection between LCFT's and critical gravity was discovered in three dimensions, it was found to hold also in higher-dimensional higher-derivative gravity theories [11-15]. The mechanism by which logarithmic modes appear in the theory is similar to the three-dimensional case. At the critical point, a degeneracy takes place and the massive gravitons coincide with either massless gravitons or pure gauge modes. Instead of the massive gravitons, an equal amount of logarithmic modes appears in the theory.

In this paper, we wish to combine the two recent developments described above, i.e. non-relativistic gauge-gravity duality and critical gravity. To be precise, we propose a LCFT which enjoys anisotropic scale invariance. The approach we take in defining this 'non-relativistic' LCFT is through the gauge/gravity duality: the LCFT is defined by its correlation functions, which we calculate through holographic methods starting from a higher-derivative bulk theory.

Instead of looking on the gravitational side at a higher-derivative model of gravitational, i.e. spin-2, degrees of freedom, we will consider a simpler situation involving only spin-0 degrees of freedom in a fixed non-relativistic gravitational background. This model is a non-relativistic version of the model discussed in $[22,23]$ (see [24] for a finite temperature version of this model). The model consists of a scalar field configuration in a fixed AdS background with fourth-order derivative equations of motion and is conjectured to be dual to a LCFT. The higher-derivative equation of motion can be written in terms of two coupled second order equations, involving Klein-Gordon operators, by introducing an auxiliary scalar field. At the point where the mass of the two scalar fields degenerate, logarithmic modes will appear. The boundary value of this logarithmic solution sources the logarithmic partner of the dual scalar operator and defines a logarithmic conformal field theory on the boundary. The analogy with critical gravity is as follows. A priori, the two Klein-Gordon operators involve different masses and the spectrum is described by two spin-0 excitations with different masses. At the critical point, both mass parameters are equal. Just as in critical gravity, massive gravitons coincide with massless gravitons and

\footnotetext{
${ }^{1}$ See $[20,21]$ for reviews on LCFT and further references.
} 
logarithmic modes appear, here both spin-0 excitations will coincide and a new logarithmic spin-0 mode shows up, that obeys the full fourth order equation of motion, but that is not annihilated by a single Klein-Gordon operator.

Since we are interested in a non-relativistic version of the model, we consider the background spacetime to be Lifshitz instead of AdS:

$$
d s_{\mathrm{Lif}_{\mathrm{d}+1}}^{2}=L^{2}\left(\frac{1}{r^{2 z}} d t^{2}+\frac{1}{r^{2}} d r^{2}+\frac{1}{r^{2}} d x^{a} d x_{a}\right) .
$$

Here the $r$ and $x^{a}, a=1, \ldots, d-1$, are the spatial directions, $L$ is a parameter with inverse mass dimension and $z$ is the dynamical exponent. For $z=1$ we recover the relativistic AdS background. One can show that the Lifshitz spacetime has an anisotropic conformal boundary at infinity which can be mapped to $r=0[25,26]$. The bulk metric induces an anisotropic conformal class of metrics on the boundary, where the action of the Lifshitz symmetry group on the boundary is induced from the action of the bulk isometries. The presence of logarithmic terms in representations of the Galilean Conformal Algebra and the Schrödinger-Virasoro algebra has been discussed in [27, 28].

This paper is organized as follows. In section 2 we introduce the non-relativistic version of the model mentioned above and discuss some of its basic features. Furthermore, we give, for $z=2$, the logarithmic modes which source the logarithmic partner of the dual scalar operator. Next, in section 3 we derive the main result of this work. We use holographic renormalization to obtain the two-point functions of the dual operators in our non-relativistic model and indicate that they satisfy the defining properties of a non-relativistic LCFT. As a final result, we suggest a general structure for the two-point functions of a logarithmic conformal field theory which enjoys anisotropic scale invariance. Finally, in the conclusions we discuss a few open issues and generalizations of our work.

\section{The model}

In this section we will introduce the scalar model that shares many of the features of critical gravity theories. It is, however, much simpler to study since it deals with spin-0 instead of spin-2 degrees of freedom. In subsection 2.1 we discuss some general features of the model while in subsection 2.2 we will calculate the scalar logarithmic modes for the specific case $z=2$.

\subsection{General features}

The model under consideration consists of a scalar field $\phi_{1}$ obeying a fourth order equation of motion, given by the action of two Klein-Gordon operators on the field:

$$
\left(\square-m_{1}^{2}\right)\left(\square-m_{2}^{2}\right) \phi_{1}=0 .
$$

For $m_{1}^{2} \neq m_{2}^{2}$, the solution space of this equation is spanned by the solutions of the two second order equations, obtained by acting with only one of the two Klein-Gordon operators appearing in (2.1), i.e. the full solution space is spanned by spin-0 excitations with masses $m_{1}$ and $m_{2}$. The case where $m_{1}^{2}=m_{2}^{2}=m^{2}$ is the analog of the critical point in massive 
gravities. In this case the two Klein-Gordon operators appearing in (2.1) are degenerate and apart from a spin-0 excitation, the spectrum also contains a logarithmic mode that obeys:

$$
\left(\square-m^{2}\right)^{2} \phi^{\log }=0, \quad\left(\square-m^{2}\right) \phi^{\log } \neq 0 .
$$

In the AdS/CFT correspondence, the conformal dimension of an operator dual to a massive scalar field is related to the mass of the scalar. As in the critical limit $m_{2}^{2} \rightarrow m_{1}^{2}=m^{2}$, the mass degenerates, one expects that the operators dual to the logarithmic mode and the scalar mode with mass $m^{2}$ will have degenerate conformal dimension and form a logarithmic pair.

In the following, we will not work with the four-derivative formulation of the model. Instead, we will introduce an auxiliary scalar field $\phi_{2}$ to lower the number of derivatives from four to two. The action (for generic $m_{1}^{2}, m_{2}^{2}$ ) we will consider is given by

$$
\begin{aligned}
S=\int d^{d+1} x \sqrt{g}( & -\frac{1}{2}\left(m_{1}^{2}-m_{2}^{2}\right)\left(\partial_{\mu} \phi_{1} \partial^{\mu} \phi_{1}+m_{1}^{2} \phi_{1}^{2}\right)-\partial_{\mu} \phi_{1} \partial^{\mu} \phi_{2} \\
& \left.-m_{1}^{2} \phi_{1} \phi_{2}-\frac{1}{2} \phi_{2}^{2}\right) .
\end{aligned}
$$

Upon diagonalization this action describes two spin-0 modes with masses $m_{1}^{2}$ and $m_{2}^{2}$. The kinetic terms will have opposite signs, so the theory is always non-unitary. This is reminiscent of higher dimensional non-critical massive gravities. Upon eliminating the auxiliary field $\phi_{2}$, this action leads to the equation of motion (2.1). At the critical point $m_{1}^{2}=m_{2}^{2}=m^{2}$, the action reduces to $[22,23]$

$$
S=-\int d^{d+1} x \sqrt{g}\left(\partial_{\mu} \phi_{1} \partial^{\mu} \phi_{2}+m^{2} \phi_{1} \phi_{2}+\frac{1}{2} \phi_{2}^{2}\right) \text {. }
$$

The equations of motion are then given by

$$
\left(\square-m^{2}\right) \phi_{1}=\phi_{2}, \quad\left(\square-m^{2}\right) \phi_{2}=0,
$$

which upon elimination of $\phi_{2}$ lead to a degenerate fourth-order equation for $\phi_{1}$.

From now on, we will consider the bulk action (2.4) and equations of motion (2.5) in the background of the anisotropically scale invariant Lifshitz metric (1.1). We will assume that we can ignore the backreaction of the massive scalar on the metric. This assumption is justified when the scalar field equations decouple from the metric equations of motion at least asymptotically up to the order of coefficients that contribute to the divergent terms in the bulk action [29].

To find the non-singular bulk field configurations $\phi_{i}(r, t, \mathbf{x})$, with $i=1,2$, for any smooth boundary value $\phi_{i(0)}(t, \mathbf{x})$ we need to find the bulk-to-boundary propagators $G_{i j}\left(r, t, \mathbf{x} ; 0, t^{\prime}, \mathbf{x}^{\prime}\right)$, so that:

$$
\phi_{i}(r, t, \mathbf{x})=\sum_{j=1}^{2} \int d^{d-1} \mathbf{x}^{\prime} d t^{\prime} \phi_{j(0)}\left(t^{\prime}, \mathbf{x}^{\prime}\right) G_{i j}\left(r, t, \mathbf{x} ; 0, t^{\prime}, \mathbf{x}^{\prime}\right) .
$$


It is convenient to work in Fourier space, where we transform $t$ into $\omega$ and $\mathbf{x}$ into $\mathbf{k}$. Now eq. (2.6) reads:

$$
\phi_{i}(r, \omega, \mathbf{k})=\sum_{j=1}^{2} \phi_{j(0)}(\omega, \mathbf{k}) G_{i j}(r, \omega, \mathbf{k})
$$

The bulk to boundary propagators $G_{i j}(r, \omega, \mathbf{k})$ satisfy the differential equations, for $r \neq 0$, :

$$
\begin{aligned}
& \left(\square-m^{2}\right) G_{22}=0, \\
& \left(\square-m^{2}\right) G_{21}=0, \\
& \left(\square-m^{2}\right) G_{11}=G_{21} \text {, } \\
& \left(\square-m^{2}\right) G_{12}=G_{22} \text {, }
\end{aligned}
$$

with

$$
\begin{aligned}
& \left(\square-m^{2}\right) G(r, \omega, \mathbf{k}) \\
& =r^{2} \partial_{r}^{2} G(r, \omega, \mathbf{k})-(d+z-2) r \partial_{r} G(r, \omega, \mathbf{k})-\left(r^{2 z} \omega^{2}+r^{2}|\mathbf{k}|^{2}+m^{2}\right) G(r, \omega, \mathbf{k}) .
\end{aligned}
$$

We have set $L=1$ for convenience. This parameter can always be re-introduced by dimensional analysis.

We note that $\phi_{1}$ is the fundamental field that satisfies a degenerate fourth-order equation of motion whereas $\phi_{2}$ is an auxiliary field, needed to rewrite the equation of motion in terms of a second-order differential equation. The most general solution for $\phi_{1}$ is therefore a superposition of a mode annihilated by acting on it with the Klein-Gordon operator once (the scalar mode) and a mode annihilated by acting twice with the Klein-Gordon operator (the logarithmic mode). Writing out eq. (2.7) for $\phi_{1}$ we have now two options. Either $\phi_{1(0)} G_{11}$ is the scalar mode and $\phi_{2(0)} G_{12}$ the logarithmic mode or vice versa. These two options correspond to the freedom we have in coupling the sources to the dual operators. We can either choose to couple $\phi_{1(0)}$ to the scalar operator and $\phi_{2(0)}$ to its logarithmic partner or vice versa. There is no difference in the physics between the two options. We fix this ambiguity by taking $G_{11}=G_{22}=G$ and $G_{21}=0$ so that eq. (2.7) becomes:

$$
\begin{aligned}
& \phi_{1}(r, \omega, \mathbf{k})=\phi_{1(0)}(\omega, \mathbf{k}) G(r, \omega, \mathbf{k})+\phi_{2(0)}(\omega, \mathbf{k}) G_{12}(r, \omega, \mathbf{k}), \\
& \phi_{2}(r, \omega, \mathbf{k})=\phi_{2(0)}(\omega, \mathbf{k}) G(r, \omega, \mathbf{k}) .
\end{aligned}
$$

Acting with one Klein-Gordon operator on $\phi_{1}$ will annihilate the $\phi_{1(0)} G$ term. This term therefore represents the scalar mode. The remaining $\left(\square-m^{2}\right) \phi_{2(0)} G_{12}$ term is equal to $\phi_{2}$ and consequently is eliminated by acting on it with a second Klein-Gordon operator. Therefore, this term represents the logarithmic mode. From the above it is clear that $\phi_{1(0)}$ couples to a scalar operator $\mathcal{O}_{\Delta}^{s}$ and that $\phi_{2(0)}$ couples to its logarithmic partner $\mathcal{O}_{\Delta}^{\log }$ where $\Delta$ is the common conformal dimension of the two operators.

The bulk-to-boundary propagator generally has two independent solutions. These solutions can be divided into modes which are regular in the interior (for $r \rightarrow \infty$ ) and singular modes. Since the singular modes diverge rapidly in the interior, it is no longer safe to assume that their backreaction to the metric can be ignored. All singular modes will therefore be discarded. 
An expansion of the field near the boundary $(r \rightarrow 0)$ allows us to also distinguish between the non-normalizable modes $\phi_{i(0)}$ and the normalizable modes $\tilde{\phi}_{i(0)}$ :

$$
\phi_{i}(r, \omega, \mathbf{k})=\phi_{i(0)}(\omega, \mathbf{k}) r^{\Delta_{-}}(1+\cdots)+\tilde{\phi}_{i(0)}(\omega, \mathbf{k}) r^{\Delta_{+}}(1+\cdots),
$$

where the dots indicate higher powers of $r$ within the brackets and $\Delta_{+} \geq \Delta_{-}$are the two roots of the quadratic equation

$$
\Delta(\Delta-(d+z-1))=m^{2}
$$

i.e.

$$
\Delta_{ \pm}=\frac{1}{2}\left((d+z-1) \pm \sqrt{(d+z-1)^{2}+4 m^{2}}\right) .
$$

Note that by requiring that $\Delta_{+} \geq \Delta_{-}$we are assuming that $\Delta_{+} \geq(d+z-1) / 2$. According to the standard AdS/CFT dictionary the non-normalizable mode $\phi_{i(0)}$ is the source for the dual field theory operator, while the normalizable mode $\tilde{\phi}_{i(0)}$ is related to the one-point function of the dual operator with conformal weight $\Delta=\Delta_{+}$.

Since the conformal dimension is related to the mass of the scalar field in the bulk, the limit where the mass of the scalar fields $\phi_{1}$ and $\phi_{2}$ degenerates corresponds to a degenerate conformal dimension for the dual operators. This is precisely what we need for a logarithmic conformal field theory, since operators with a degenerate conformal dimension will form a logarithmic pair with a non-diagonalizable Jordan cell. This degeneracy should not be confused with the degeneracy between $\Delta_{+}$and $\Delta_{-}$plus even integers [30] (see [31] for the non-relativistic extension). We will comment briefly here on this kind of degeneracies.

The form of the power series in eq. (2.13) can be determined by solving the equations of motion order by order in $r$. In our case, it is an expansion in $r^{2 k}$ and $r^{2 z l}$, with $k, l \in \mathbb{Z}$. Therefore, whenever $\Delta_{+}-\Delta_{-}$is an even integer or a multiple of $2 z$, the corresponding term in the expansion of $\Delta_{-}$will degenerate with the leading term in the expansion of $\Delta_{+}$and a logarithmic term needs to be introduced at order $r^{\Delta_{+}}$. We can relate this to a value of the scalar field mass as follows:

$$
\Delta_{+}-\Delta_{-}=\sqrt{(d+z-1)^{2}+4 m^{2}}=2(k+l z), \quad k, l \in \mathbb{Z} .
$$

The special case where $\Delta_{+}=\Delta_{-}=(d+z-1) / 2$ saturates the Breitenlohner-Freedman bound

$$
m^{2} \geq-(d+z-1)^{2} / 4
$$

In that case the asymptotic expansion acquires a logarithmic term at leading order, because the two $\Delta$ 's degenerate:

$$
\phi_{i}(r, \omega, \mathbf{k})=r^{\Delta}\left(\phi_{i(0)}(\omega, \mathbf{k})+\cdots+\log (r)\left(\tilde{\phi}_{i(0)}(\omega, \mathbf{k})+\cdots\right)\right) .
$$

In the presence of this kind of degeneracies, one needs to take additional logarithmic counterterms into account in order to get finite correlation functions. Analogous to the discussions in [30], this will result in a term in the one-point function which is a local 
function of the sources. At the level of the higher-point functions these will correspond to contact terms. For the sake of simplicity we will restrict ourselves to those values of $\mathrm{m}^{2}$ for which no logarithmic terms arise in the expansion of $\phi_{i}$ due to this kind of degeneracies. In this work we only consider the consequences of the degeneracy of the scalar field masses $m_{1}$ and $m_{2}$. Therefore, the results presented in section 3 hold for general $m^{2}$ only up to contact terms in the two-point correlation functions.

\subsection{An example: $z=2$}

To find an explicit expression for the logarithmic mode we first need to find an exact solution for the scalar mode. Such a solution is available for the case $z=2[2]$. We therefore consider that example in this subsection. The solution of the homogeneous Klein-Gordon equation (2.8) with $G_{11}=G_{22}=G$ and $G_{21}=0$ is given by:

$$
G(r, \omega, \mathbf{k}) \propto r^{\Delta} e^{-\frac{1}{2} \omega r^{2}} U\left(\frac{|\mathbf{k}|^{2}+(2 \Delta-(d-1)) \omega}{4 \omega}, \Delta-\frac{d-1}{2}, \omega r^{2}\right),
$$

where we now have that:

$$
\Delta=\frac{1}{2}\left(d+1+\sqrt{(d+1)^{2}+4 m^{2}}\right) .
$$

$U(a, b, x)$ is the confluent hypergeometric function and the constant of proportionality can be determined by requiring that $G(\epsilon, \omega, \mathbf{k})=1$ on the regulated boundary $r=\epsilon$. We have found $\phi_{2}(r, \omega, \mathbf{k})$

$$
\phi_{2}(r, \omega, \mathbf{k})=\phi_{2(0)}(\omega, \mathbf{k}) G(r, \omega, \mathbf{k}),
$$

which can be expanded near the boundary as:

$$
\begin{aligned}
\phi_{2}(r, \omega, \mathbf{k})= & \phi_{2(0)}(\omega, \mathbf{k}) r^{d+1-\Delta}[1+\cdots] \\
& +\phi_{2(0)}(\omega, \mathbf{k}) \frac{\Gamma\left(\frac{d+1}{2}-\Delta\right) \Gamma\left(\frac{|\mathbf{k}|^{2}+(2 \Delta-(d-1)) \omega}{4 \omega}\right)}{\Gamma\left(\Delta-\frac{d+1}{2}\right) \Gamma\left(\frac{|\mathbf{k}|^{2}-(2 \Delta-(d+3)) \omega}{4 \omega}\right)} \omega^{\Delta-\frac{d+1}{2}} r^{\Delta}[1+\cdots] .
\end{aligned}
$$

Now we still need to find $G_{12}$. For this we use a trick inspired by [23]. The equation which determines $G_{12}$ is:

$$
\left(\square-m^{2}\right) G_{12}(r, \omega, \mathbf{k})=G(r, \omega, \mathbf{k}) .
$$

From eq. (2.14) it follows that $\left[\left(\square-m^{2}\right), d / d \Delta\right]=d m^{2} / d \Delta=2 \Delta-(d+1)$ where we have used that the Lifshitz metric does not depend on the conformal dimension $\Delta$. Using that $\left(\square-m^{2}\right) G(r, \omega, \mathbf{k})=0$ we can therefore write $G$ as:

$$
G=\frac{1}{2 \Delta-(d+1)}\left[\left(\square-m^{2}\right), \frac{d}{d \Delta}\right] G=\frac{1}{(2 \Delta-(d+1))}\left(\square-m^{2}\right) \frac{d}{d \Delta} G .
$$

Comparing this with eq. (2.23) we derive the following expression of $G_{12}$ in terms of the derivative of $G$ with respect to $\Delta:^{2}$

$$
G_{12}(r, \omega, \mathbf{k})=\frac{1}{2 \Delta-(d+1)} \frac{d}{d \Delta} G(r, \omega, \mathbf{k}) .
$$

\footnotetext{
${ }^{2}$ Note that this method is identical to the method employed in $[5,6]$ to find the log modes of TMG at the critical point, albeit adjusted for scalar fields. Here one takes the limit $m_{1}^{2} \rightarrow m_{2}^{2}$ of $\left(\phi_{1}\left(m_{1}^{2}\right)-\right.$ $\left.\phi_{2}\left(m_{2}^{2}\right)\right) /\left(m_{1}^{2}-m_{2}^{2}\right)$.
} 
The derivative of the confluent hypergeometric function is not so easy to find. However, for our purposes, it is sufficient to derive the near boundary expansion of this derivative. The expression for this expansion can be found by taking the derivative of the expansion (2.22).

According to eq. (2.11) we have that

$$
\phi_{1}(r, \omega, \mathbf{k})=\phi_{1(0)}(\omega, \mathbf{k}) G(r, \omega, \mathbf{k})+\phi_{2(0)}(\omega, \mathbf{k}) \frac{1}{2 \Delta-(d+1)} \frac{d G(r, \omega, \mathbf{k})}{d \Delta} .
$$

This finally leads to the following near-boundary expansion for $\phi_{1}$ :

$$
\begin{aligned}
\phi_{1}(r, \omega, \mathbf{k})= & \left(\phi_{1(0)}(\omega, \mathbf{k})+\phi_{2(0)}(\omega, \mathbf{k}) \frac{1}{((d+1)-2 \Delta)} \log (r)\right) r^{d+1-\Delta}[1+\cdots] \\
+ & \left(\phi_{1(0)}(\omega, \mathbf{k})-\phi_{2(0)}(\omega, \mathbf{k}) \frac{1}{(d+1-2 \Delta)}(\log (r)+\log (\omega)\right. \\
& \quad-\psi\left(\frac{d+1}{2}-\Delta\right)-\psi\left(\Delta-\frac{d+1}{2}\right)+\frac{1}{2} \psi\left(\frac{|\mathbf{k}|^{2}+(2 \Delta-(d-1)) \omega}{4 \omega}\right) \\
& \left.\left.\quad+\frac{1}{2} \psi\left(\frac{|\mathbf{k}|^{2}-(2 \Delta-(d+3)) \omega}{4 \omega}\right)\right)\right) \\
& \times \frac{\Gamma\left(\frac{d+1}{2}-\Delta\right) \Gamma\left(\frac{|\mathbf{k}|^{2}+(2 \Delta-(d-1)) \omega}{4 \omega}\right)}{\Gamma\left(\Delta-\frac{d+1}{2}\right) \Gamma\left(\frac{|\mathbf{k}|^{2}-(2 \Delta-(d+3)) \omega}{4 \omega}\right)} \omega^{\Delta-\frac{d+1}{2}} r^{\Delta}[1+\cdots]
\end{aligned}
$$

where $\psi(x)$ is the digamma function defined by $\psi(x)=\Gamma^{\prime}(x) / \Gamma(x)$.

Note that there is a shift symmetry in the expression for $\phi_{1}$. We can always add solutions to the homogeneous Klein-Gordon equation to $\phi_{1}$ and it will still solve the field equations. This is due to a symmetry of the action (2.4). The variation in the action under the transformation $\phi_{1} \rightarrow \phi_{1}+\lambda \phi_{2}, \phi_{2} \rightarrow \phi_{2}$ with constant $\lambda$ is proportional to the equation of motion and zero on-shell. At the level of two-point functions of the dual theory, which we will calculate in the next section, this shift symmetry corresponds to a well-known ambiguity in defining the logarithmic operator.

\section{Two point correlation functions}

Having obtained, for a specific example, the explicit expression for the logarithmic modes we now proceed to relate these solutions to operators on the boundary of the Lifshitz spacetime. For this we need to apply the holographic renormalization procedure [30]. In subsection 3.1 we first briefly review some aspects of this procedure which will be needed later on. Next, in subsection 3.2, we will calculate the two-point correlation functions for an AdS background, i.e. $z=1$ and for the example discussed in subsection 2.2, i.e. $z=2$. We will show that in both examples the two-point functions satisfy the defining properties of a relativistic and non-relativistic LCFT, respectively.

\subsection{Holographic renormalization}

For the purpose of this subsection we may switch back to general values of $z$. Only in the next subsection we will specify this value. Following the AdS/CFT correspondence, we 
couple the boundary values of the scalar field to operators in the field theory:

$$
\int d^{d-1} x d t\left(\phi_{1(0)} \mathcal{O}_{\Delta}^{\mathrm{s}}+\beta \phi_{2(0)} \mathcal{O}_{\Delta}^{\log }\right)
$$

where $\beta$ is a normalization parameter which we will fix later on. To precisely compute the two-point function we need to get rid of the divergences in the bulk fields as we move towards the boundary. We can do so by means of a holographic renormalization of the action (2.4). Following [30] we first compute the on-shell action $S_{\text {reg }}$ on a regulated surface $r=\epsilon$, using a near boundary expansion of the fields. Then we identify the divergent terms in this action as a function of the sources $\phi_{i(0)}$ and write down the counterterm action $S_{\mathrm{ct}}$ as minus these divergent terms. The counterterm action cannot be written as a covariant expression; it obeys the same anisotropic scaling as the Lifshitz background. Of course the limit $z \rightarrow 1$ should reduce to the AdS results which does allow a covariant expression. Once the counterterm action is obtained, this can be subtracted at the regulated surface to obtain the subtracted action $S_{\text {sub }}$ which has by construction a finite limit for $\epsilon \rightarrow 0$.

Following the AdS/CFT dictionary, the one-point correlation functions can be obtained by functional differentiation of the on-shell action with respect to the sources:

$$
\left\langle\mathcal{O}_{\Delta}^{i}(t, \mathbf{x})\right\rangle=\left.\frac{\delta S_{\mathrm{sub}}}{\delta \phi_{i(0)}(t, \mathbf{x})}\right|_{\phi_{i(0)}=0} .
$$

Since the subtracted action is expressed in terms of the bulk fields $\phi_{1}$ and $\phi_{2}$ on the regulated boundary, we need to write the above expression for the one-point correlation functions in terms of derivatives with respect to the bulk fields and afterwards take the limit $\epsilon \rightarrow 0$. To rewrite sources in terms of bulk fields we consider the near-boundary expansions of the bulk fields $\phi_{1}$ and $\phi_{2}$ :

$$
\begin{aligned}
\phi_{1}= & \left(\phi_{1(0)}+\alpha \phi_{2(0)} \log r\right) r^{d+z-1-\Delta}+\left(\phi_{1(2)}+\alpha \phi_{2(2)} \log r\right) r^{d+z+1-\Delta} \\
& +\left(\phi_{1(2 z)}+\alpha \phi_{2(2 z)} \log r\right) r^{d+3 z-1-\Delta}+\cdots+\left(\tilde{\phi}_{1(0)}-\alpha \tilde{\phi}_{2(0)} \log r\right) r^{\Delta}+\cdots \\
\phi_{2}= & \phi_{2(0)} r^{d+z-1-\Delta}+\phi_{2(2)} r^{d+z+1-\Delta}+\phi_{2(2 z)} r^{d+3 z-1-\Delta}+\cdots+\tilde{\phi}_{2(0)} r^{\Delta}+\cdots,
\end{aligned}
$$

where $\alpha$ is given by

$$
\alpha=\frac{1}{(d+z-1-2 \Delta)} .
$$

We can use the leading order terms in this expansion to write (3.2) in terms of a functional derivative with respect to the bulk fields $\phi_{1}, \phi_{2}$ :

$$
\begin{aligned}
\left\langle\mathcal{O}_{\Delta}^{\mathrm{s}}(t, \mathbf{x})\right\rangle & =\lim _{\epsilon \rightarrow 0}\left(\frac{1}{\sqrt{\gamma}} \frac{1}{\epsilon^{\Delta}} \frac{\delta S_{\mathrm{sub}}}{\delta \phi_{1}(\epsilon, t, \mathbf{x})}\right) \\
\beta\left\langle\mathcal{O}_{\Delta}^{\log }(t, \mathbf{x})\right\rangle & =\lim _{\epsilon \rightarrow 0}\left(\frac{1}{\sqrt{\gamma}} \frac{1}{\epsilon^{\Delta}}\left(\frac{\delta S_{\mathrm{sub}}}{\delta \phi_{2}(\epsilon, t, \mathbf{x})}+\alpha \log \epsilon \frac{\delta S_{\mathrm{sub}}}{\delta \phi_{1}(\epsilon, t, \mathbf{x})}\right)\right),
\end{aligned}
$$

where $\gamma_{\alpha \beta} d x^{\alpha} d x^{\beta}=d x_{a} d x^{a} / \epsilon^{2}+d t^{2} / \epsilon^{2 z}$ is the induced metric on the regulated hypersurface and $\gamma$ is its determinant. 
The two-point functions are obtained by a further differentiation of the one-point functions with respect to the sources and setting the sources to zero afterwards :

$$
\begin{aligned}
\left\langle\mathcal{O}_{\Delta}^{\mathrm{s}}(t, \mathbf{x}) \mathcal{O}_{\Delta}^{\mathrm{s}}\left(t_{2}, \mathbf{x}_{2}\right)\right\rangle & =-\left.\frac{\delta\left\langle\mathcal{O}_{\Delta}^{\mathrm{s}}(t, \mathbf{x})\right\rangle}{\delta \phi_{1(0)}\left(t_{2}, \mathbf{x}_{2}\right)}\right|_{\phi_{1(0)}=0}, \\
\beta\left\langle\mathcal{O}_{\Delta}^{\log }(t, \mathbf{x}) \mathcal{O}_{\Delta}^{\mathrm{s}}\left(t_{2}, \mathbf{x}_{2}\right)\right\rangle & =-\left.\frac{\delta\left\langle\mathcal{O}_{\Delta}^{\mathrm{s}}(t, \mathbf{x})\right\rangle}{\delta \phi_{2(0)}\left(t_{2}, \mathbf{x}_{2}\right)}\right|_{\phi_{2(0)}=0}=-\left.\frac{\delta\left\langle\mathcal{O}_{\Delta}^{\log }(t, \mathbf{x})\right\rangle}{\delta \phi_{1(0)}\left(t_{2}, \mathbf{x}_{2}\right)}\right|_{\phi_{1(0)}=0}, \\
\beta^{2}\left\langle\mathcal{O}_{\Delta}^{\log }(t, \mathbf{x}) \mathcal{O}_{\Delta}^{\log }\left(t_{2}, \mathbf{x}_{2}\right)\right\rangle & =-\left.\frac{\delta\left\langle\mathcal{O}_{\Delta}^{\log }(t, \mathbf{x})\right\rangle}{\delta \phi_{2(0)}\left(t_{2}, \mathbf{x}_{2}\right)}\right|_{\phi_{2(0)}=0} .
\end{aligned}
$$

We now apply the holographic renormalization procedure to the scalar model defined by the action (2.4). A partial integration of this action on a regulated surface $r=\epsilon$ near the boundary and requiring the equations of motion to hold leads to the following regularized on-shell action:

$$
S_{\mathrm{reg}}=-\frac{1}{2} \int_{r=\epsilon} d^{d} x \sqrt{\gamma}\left(\phi_{1} \vec{n} \cdot \vec{\nabla} \phi_{2}+\phi_{2} \vec{n} \cdot \vec{\nabla} \phi_{1}\right),
$$

where $\vec{n}$ is the vector normal to the regulated hypersurface $\vec{n} \cdot \vec{\nabla}=\left.r \partial_{r}\right|_{r=\epsilon}$.

Without explicitly going through all the steps of the holographic renormalization procedure, we note that after a lengthy calculation we find that the counterterm action needed to make the action (2.4) finite is given by:

$$
\begin{aligned}
S_{\mathrm{ct}}=\int_{r=\epsilon} d^{d} x \sqrt{\gamma}( & (d+z-1-\Delta) \phi_{1} \phi_{2}+\frac{1}{2} \alpha \phi_{2} \phi_{2} \\
& -a_{2}\left(\frac{1}{2}\left(\phi_{1} \partial^{a} \partial_{a} \phi_{2}+\phi_{2} \partial^{a} \partial_{a} \phi_{1}\right)-a_{2} \alpha \phi_{2} \partial^{a} \partial_{a} \phi_{2}\right) \\
& \left.-a_{2 z}\left(\frac{1}{2}\left(\phi_{1} \partial^{t} \partial_{t} \phi_{2}+\phi_{2} \partial^{t} \partial_{t} \phi_{1}\right)-a_{2 z} \alpha \phi_{2} \partial^{t} \partial_{t} \phi_{2}\right)+\mathcal{O}\left(\phi_{i} \partial_{a}^{4} \phi_{i}\right)\right)
\end{aligned}
$$

with $a=1, \ldots, d-1$ and $a_{2}$ and $a_{2 z}$ given by ${ }^{3}$

$$
a_{2}=\frac{1}{(d+z+1-2 \Delta)}, \quad a_{2 z}=\frac{1}{(d+3 z-1-2 \Delta)} .
$$

We note that all indices in the derivatives are raised and lowered with the induced metric on the boundary $\gamma_{\alpha \beta}$.

In (3.12) we took terms up to order $\mathcal{O}\left(\phi_{i} \partial_{a}^{4} \phi_{i}\right)$ into account. In the near-boundary expansions (3.3), (3.4) the normalizable modes are of order $\epsilon^{\Delta}$, so all the terms with a lower power than $\epsilon^{\Delta}$ are going to contribute to the counterterm action. The precise

\footnotetext{
${ }^{3}$ Note that for $d=z=2$ and $\Delta=5 / 2$ a pole appears in $a_{2}$ and $a_{2 z}$. At this value of the mass $m^{2}$ the degeneracy mentioned at the end of section 2.1 takes place. The correct way to deal with these poles is to introduce additional logarithmic terms in the near-boundary expansion and replace the corresponding term in $S_{\text {ct }}$ with a $\log \epsilon$ divergent term. This will change the two-point correlation functions with contact terms. But as mentioned before, we will not explicitly take these special values of $m^{2}$ into account.
} 
number of counterterms we need to add depends on the value of $\Delta$. This value of $\Delta$ is restricted as follows:

$$
\frac{1}{2}(d+z-1) \leq \Delta \leq d+z-1 .
$$

The upper limit follows from the observation that if $\Delta \geq d+z-1$ then the operator is irrelevant and, according to [32], it is no longer safe to ignore the backreaction of the scalar sector on the gravitational background. The lower limit follows from the BreitenlohnerFreedman bound (2.17). For our purposes, taking counterterms into account up to order $\epsilon^{d+z-1}$ is sufficient. In eq. (3.12) we have only written down the first couple of terms. These are sufficient for $d=2$ and $z=1,2$. These terms illustrate that the counterterm action cannot be written down covariantly, but instead respects the anisotropic scale invariance. For larger values of $d$ and $z$ we need to take more counterterms into account, but the analysis is similar and can be extended straightforwardly. The renormalized one-point correlation functions do not change as long as the degeneracy discussed at the end of section 2.1 is absent. Their expressions are given by:

$$
\begin{aligned}
\left\langle\mathcal{O}_{\Delta}^{\mathrm{s}}(t, \mathbf{x})\right\rangle & =(d+z-1-2 \Delta) \tilde{\phi}_{2(0)}(t, \mathbf{x}), \\
\beta\left\langle\mathcal{O}_{\Delta}^{\log }(t, \mathbf{x})\right\rangle & =(d+z-1-2 \Delta) \tilde{\phi}_{1(0)}(t, \mathbf{x}),
\end{aligned}
$$

where $\beta$ is the normalization parameter that appeared in (3.1).

\subsection{Two-point correlation functions}

The two-point functions can now be obtained from the exact solutions to the field equations. Once the exact solution is found, we can expand it near the boundary and find the expressions for $\tilde{\phi}_{i(0)}$ linearly in the sources $\phi_{i(0)}$. To find exact solutions we need to specify the value of $z$. Below we discuss two examples.

\subsubsection{Example 1: $z=1$}

We first consider $z=1$, i.e. the $d$ dimensional LCFT dual to $d+1$ dimensional Anti-de Sitter. The solution to the homogeneous Klein-Gordon equation which is regular everywhere in the interior in Fourier space is:

$$
G(r, k) \propto r^{\frac{d}{2}} K_{\frac{1}{2} \sqrt{d^{2}+4 m^{2}}}(|k| r),
$$

where $k=\{\omega, \mathbf{k}\}$ is now a $d$ component vector with length $|k|$ and $K_{n}(z)$ is the modified Bessel function of the second kind. The constant of proportionality is determined by taking $G(\epsilon, k)=1$ on the regulated boundary.

Repeating the steps outlined in section 2.2 and applying the holographic renormalization outlined above for $z=1$ we find that the correlation functions expressed in Fourier space are:

$$
\begin{aligned}
\left\langle\mathcal{O}_{\Delta}^{\mathrm{s}}(k) \mathcal{O}_{\Delta}^{\mathrm{s}}(-k)\right\rangle & =0, \\
\beta\left\langle\mathcal{O}_{\Delta}^{\mathrm{s}}(k) \mathcal{O}_{\Delta}^{\log }(-k)\right\rangle & =(2 \Delta-d)|k|^{2 \Delta-d} \frac{2^{d-2 \Delta} \Gamma\left(\frac{d}{2}-\Delta\right)}{\Gamma\left(\Delta-\frac{d}{2}\right)}
\end{aligned}
$$




$$
\begin{aligned}
\beta^{2}\left\langle\mathcal{O}_{\Delta}^{\log }(k) \mathcal{O}_{\Delta}^{\log }(-k)\right\rangle=|k|^{2 \Delta-d} & \frac{2^{d-2 \Delta} \Gamma\left(\frac{d}{2}-\Delta\right)}{\Gamma\left(\Delta-\frac{d}{2}\right)}(2 \log |k| \\
& \left.-\log 4-\psi\left(\Delta-\frac{d}{2}\right)-\psi\left(\frac{d}{2}-\Delta\right)+\lambda\right) .
\end{aligned}
$$

As expected, this is precisely the structure of a relativistic LCFT [23]. The constant $\lambda$ in the last correlator above is a consequence of the shift symmetry $\phi_{1} \rightarrow \phi_{1}+\lambda \phi_{2}$ of the original (on-shell) action. It corresponds to a well known ambiguity in LCFT's, where one can always redefine the logarithmic operator by the shift $\mathcal{O}^{\log } \rightarrow \mathcal{O}^{\log }+\lambda^{\prime} \mathcal{O}^{\text {s }}$.

\subsubsection{Example 2: $z=2$}

For the example worked out in subsection 2.2 with $z=2$ we can read off $\tilde{\phi}_{1(0)}$ and $\tilde{\phi}_{2(0)}$ by comparing (3.3) with (2.22) and (3.4) with (2.27). This leads to the following expressions:

$$
\begin{aligned}
\tilde{\phi}_{1(0)}=\omega^{\Delta-} \frac{d+1}{2} & \frac{\Gamma\left(\frac{d+1}{2}-\Delta\right) \Gamma\left(\frac{|\mathbf{k}|^{2}+(2 \Delta-(d-1)) \omega}{4 \omega}\right)}{\Gamma\left(\Delta-\frac{d+1}{2}\right) \Gamma\left(\frac{|\mathbf{k}|^{2}-(2 \Delta-(d+3)) \omega}{4 \omega}\right)}\left(\phi_{1(0)}-\phi_{2(0)} \frac{1}{d+1-2 \Delta}(\log (\omega)\right. \\
- & \left(\frac{d+1}{2}-\Delta\right)-\psi\left(\Delta-\frac{d+1}{2}\right)+\frac{1}{2} \psi\left(\frac{|\mathbf{k}|^{2}+(2 \Delta-(d-1)) \omega}{4 \omega}\right) \\
& \left.\left.+\frac{1}{2} \psi\left(\frac{|\mathbf{k}|^{2}-(2 \Delta-(d+3)) \omega}{4 \omega}\right)\right)\right) \\
\tilde{\phi}_{2(0)}= & \phi_{2(0)} \frac{1}{d+1-2 \Delta} \omega^{\Delta-\frac{d+1}{2}} \frac{\Gamma\left(\frac{d+1}{2}-\Delta\right) \Gamma\left(\frac{|\mathbf{k}|^{2}+(2 \Delta-(d-1)) \omega}{4 \omega}\right)}{\Gamma\left(\Delta-\frac{d+1}{2}\right) \Gamma\left(\frac{|\mathbf{k}|^{2}-(2 \Delta-(d+3)) \omega}{4 \omega}\right)} .
\end{aligned}
$$

Following the general procedure outlined in the previous subsection we find that the twopoint functions are:

$$
\begin{aligned}
\left\langle\mathcal{O}_{\Delta}^{\mathrm{s}}(\omega, \mathbf{k}) \mathcal{O}_{\Delta}^{\mathrm{s}}(-\omega,-\mathbf{k})\right\rangle= & , \\
\beta\left\langle\mathcal{O}_{\Delta}^{\mathrm{s}}(\omega, \mathbf{k}) \mathcal{O}_{\Delta}^{\log }(-\omega,-\mathbf{k})\right\rangle= & (2 \Delta-(d+1)) \omega^{\Delta-\frac{d+1}{2}} \frac{\Gamma\left(\frac{d+1}{2}-\Delta\right) \Gamma\left(\frac{|\mathbf{k}|^{2}+(2 \Delta-(d-1)) \omega}{4 \omega}\right)}{\Gamma\left(\Delta-\frac{d+1}{2}\right) \Gamma\left(\frac{|\mathbf{k}|^{2}-(2 \Delta-(d+3)) \omega}{4 \omega}\right)} \\
\beta^{2}\left\langle\mathcal{O}_{\Delta}^{\log }(\omega, \mathbf{k}) \mathcal{O}_{\Delta}^{\log }(-\omega,-\mathbf{k})\right\rangle= & \omega^{\Delta-\frac{d+1}{2}} \frac{\Gamma\left(\frac{d+1}{2}-\Delta\right) \Gamma\left(\frac{|\mathbf{k}|^{2}+(2 \Delta-(d-1)) \omega}{4 \omega}\right)}{\Gamma\left(\Delta-\frac{d+1}{2}\right) \Gamma\left(\frac{|\mathbf{k}|^{2}-(2 \Delta-(d+3)) \omega}{4 \omega}\right)}(\log \omega \\
& -\psi\left(\frac{d+1}{2}-\Delta\right)-\psi\left(\Delta-\frac{d+1}{2}\right) \\
& +\frac{1}{2} \psi\left(\frac{|\mathbf{k}|^{2}+(2 \Delta-(d-1)) \omega}{4 \omega}\right) \\
& \left.+\frac{1}{2} \psi\left(\frac{|\mathbf{k}|^{2}-(2 \Delta-(d+3)) \omega}{4 \omega}\right)+\lambda\right)
\end{aligned}
$$

The correlation function (3.24) agrees with the two point function for a massive scalar field in a Lifshitz background found in [2] and later by means of holographic renormalization in [31]. 


\subsection{Comparison with LCFT's}

In the relativistic case, a general logarithmic conformal field theory of rank 2 (i.e. only one logarithmic partner) has two-point correlation functions which are restricted by the conformal symmetry to be [19]:

$$
\begin{aligned}
\left\langle\mathcal{O}^{\mathrm{s}}(x) \mathcal{O}^{\mathrm{S}}(y)\right\rangle & =0, \\
\left\langle\mathcal{O}^{\log }(x) \mathcal{O}^{\mathrm{S}}(y)\right\rangle & =\frac{c}{|x-y|^{2 \Delta}}, \\
\left\langle\mathcal{O}^{\log }(x) \mathcal{O}^{\log }(y)\right\rangle & =\frac{1}{|x-y|^{2 \Delta}}(-2 c \log |x-y|+\lambda),
\end{aligned}
$$

where the constant $c$ is determined by the normalization of $\mathcal{O}^{\log }$ and the constant $\lambda$ can be changed by shifting $\mathcal{O}^{\log } \rightarrow \mathcal{O}^{\log }+\lambda^{\prime} \mathcal{O}^{\text {s }}$.

To re-write these expressions in Fourier space we use the fact that the Fourier transform of a power law in $d$ dimensions is given by another power law. Explicitly, one finds:

$$
\begin{aligned}
\left\langle\mathcal{O}^{\log }(k) \mathcal{O}^{\mathrm{s}}(-k)\right\rangle & =\frac{1}{(2 \pi)^{d / 2}} \int d^{d} x e^{-i k \cdot x} \frac{c}{|x|^{2 \Delta}} \\
& =2^{d / 2-2 \Delta} \frac{\Gamma\left(\frac{d}{2}-\Delta\right)}{\Gamma\left(\frac{d}{2}\right) \Gamma\left(1+\Delta-\frac{d}{2}\right)} c|k|^{2 \Delta-d}
\end{aligned}
$$

and:

$$
\begin{aligned}
\left\langle\mathcal{O}^{\log }(k) \mathcal{O}^{\log }(-k)\right\rangle= & \frac{1}{(2 \pi)^{d / 2}} \int d^{d} x e^{-i k \cdot x} \frac{c}{|x|^{2 \Delta}}(-2 \log x+\lambda) \\
= & 2^{d / 2-2 \Delta-1} \frac{\Gamma\left(\frac{d}{2}-\Delta\right)}{\Gamma\left(\frac{d}{2}\right) \Gamma\left(1+\Delta-\frac{d}{2}\right)} c|k|^{2 \Delta-d}(2 \log |k| \\
& \left.\quad-\log 4-\psi\left(\frac{d}{2}-\Delta\right)-\psi\left(1+\Delta-\frac{d}{2}\right)+2 \lambda\right) .
\end{aligned}
$$

If we compare these expressions with the correlation functions obtained in section 3.2.1 from the holographic calculation with bulk AdS space we find that they agree and the standard normalization is obtained by choosing $\beta=1 /(\Delta-d / 2)$.

For non-relativistic field theories the two-point functions are less restricted by the symmetry group. Invariance under time and space translations and spatial rotations restrict the two point correlation functions to be functions of only $\left|t-t^{\prime}\right|$ and $\left|\mathbf{x}-\mathbf{x}^{\prime}\right|$. The nonrelativistic scale transformations then further restrict the general two-point function of two operators with scaling dimensions $\Delta_{1}$ and $\Delta_{2}$ to be:

$$
\left\langle\mathcal{O}_{\Delta_{1}}\left(t_{1}, \mathbf{x}_{1}\right) \mathcal{O}_{\Delta_{2}}\left(t_{2}, \mathbf{x}_{2}\right)\right\rangle=\frac{1}{\left|\mathbf{x}_{1}-\mathbf{x}_{2}\right|^{\Delta_{1}+\Delta_{2}}} f(\chi)=\frac{1}{\left|t_{1}-t_{2}\right|^{\left(\Delta_{1}+\Delta_{2}\right) / z}} f^{\prime}(\chi),
$$

where $f(\chi), f^{\prime}(\chi)$ are arbitrary functions of the scale invariant variable $\chi=\frac{\left|\mathbf{x}_{1}-\mathbf{x}_{2}\right| z}{\left|t_{1}-t_{2}\right|}$.

If we compare this with the correlation functions found in section 3.2 .2 we see that they show the appropriate scaling behavior. By analogy to the AdS case, this suggests that the general structure of the non-relativistic LCFT is:

$$
\left\langle\mathcal{O}^{\mathrm{s}}\left(t_{1}, \mathbf{x}_{1}\right) \mathcal{O}^{\mathrm{s}}\left(t_{2}, \mathbf{x}_{2}\right)\right\rangle=0
$$




$$
\begin{aligned}
\left\langle\mathcal{O}^{\log }\left(t_{1}, \mathbf{x}_{1}\right) \mathcal{O}^{\mathrm{s}}\left(t_{2}, \mathbf{x}_{2}\right)\right\rangle & =\frac{1}{\left|\mathbf{x}_{1}-\mathbf{x}_{2}\right|^{2 \Delta}} f(\chi), \\
\left\langle\mathcal{O}^{\log }\left(t_{1}, \mathbf{x}_{1}\right) \mathcal{O}^{\log }\left(t_{2}, \mathbf{x}_{2}\right)\right\rangle & =\frac{1}{\left|\mathbf{x}_{1}-\mathbf{x}_{2}\right|^{2 \Delta}}\left(-g(\chi) \log \left|\mathbf{x}_{1}-\mathbf{x}_{2}\right|+\lambda\right),
\end{aligned}
$$

with $\lambda$ a constant which can be changed by transforming $\mathcal{O}^{\log } \rightarrow \mathcal{O}^{\log }+\lambda^{\prime} \mathcal{O}^{\text {s }}$ and $f(\chi), g(\chi)$ are arbitrary functions of the scale invariant variable $\chi$.

\section{Conclusions}

In this work we considered a fourth-order derivative scalar field configuration. Upon using an auxiliary scalar field, the model describes two ordinary Klein-Gordon scalar fields with mass squared $m_{1}^{2}$ and $m_{2}^{2}$ and with opposite signs of their kinetic terms. Like in theories of massive gravity, there exists a critical case where $m_{1}^{2}=m_{2}^{2}=m^{2}$, that exhibits a logarithmic mode, apart from an ordinary scalar mode. In the relativistic case, when considering a fixed AdS background, the model was shown to be dual to a logarithmic CFT [23]. Instead of considering a fixed AdS background, in this paper we considered a non-relativistic Lifshitz background. Just as the usual AdS/CFT correspondence is then extended to a non-relativistic version, likewise we suggest that the fourth-order derivative scalar model is dual to a non-relativistic version of a logarithmic CFT. We then employed non-relativistic holographic methods to calculate the two-point functions of the operators sourced by the boundary value of the scalar and the logarithmic mode. Holographic reasoning allows one to view these correlation functions on the boundary as defining a non-relativistic extension of a logarithmic CFT.

Although the model we discussed here involves only spin-0 degrees of freedom, it bears a lot of resemblance with massive gravity theories. Away from critical points, the latter describe both massive and massless (or pure gauge for $d=3$ ) spin-2 degrees of freedom. At a critical point, the massive gravitons become massless and are replaced by logarithmic modes. At such a critical point, the theories are conjectured to be dual to logarithmic CFTs. In view of this similarity to critical gravity, it would be interesting to consider critical gravities around a non-relativistic background and obtain non-relativistic versions of the $\log$ CFTs dual to massive gravity theories. In these log CFTs, typically the stress energy tensor would acquire a logarithmic partner. In this respect it is of interest to note that massive gravity theories, like Topologically Massive Gravity and New Massive Gravity, generically exhibit Lifshitz vacua.

Finally, it would be interesting to see whether these non-relativistic log CFTs, obtained via holographic reasoning can also be understood as deformations of relativistic log CFTs as it can be done for ordinary non-relativistic CFTs [33].

\section{Acknowledgments}

S.d.H, W.M. and J.R. are financed by the Dutch stichting voor Fundamenteel Onderzoek der Materie (FOM). 
Open Access. This article is distributed under the terms of the Creative Commons Attribution Noncommercial License which permits any noncommercial use, distribution, and reproduction in any medium, provided the original author(s) and source are credited.

\section{References}

[1] J.M. Maldacena, The large- $N$ limit of superconformal field theories and supergravity, Int. J. Theor. Phys. 38 (1999) 1113 [Adv. Theor. Math. Phys. 2 (1998) 231] [hep-th/9711200] [SPIRES].

[2] S. Kachru, X. Liu and M. Mulligan, Gravity duals of Lifshitz-like fixed points, Phys. Rev. D 78 (2008) 106005 [arXiv:0808.1725] [SPIRES].

[3] D.T. Son, Toward an AdS/cold atoms correspondence: a geometric realization of the Schrödinger symmetry, Phys. Rev. D 78 (2008) 046003 [arXiv: 0804.3972] [SPIRES].

[4] K. Balasubramanian and J. McGreevy, Gravity duals for non-relativistic CFTs, Phys. Rev. Lett. 101 (2008) 061601 [arXiv:0804 .4053] [SPIRES].

[5] D. Grumiller and N. Johansson, Instability in cosmological topologically massive gravity at the chiral point, JHEP 07 (2008) 134 [arXiv:0805.2610] [SPIRES].

[6] S. Ertl, D. Grumiller and N. Johansson, Erratum to 'Instability in cosmological topologically massive gravity at the chiral point', arXiv:0805.2610, arXiv:0910.1706 [SPIRES].

[7] K. Skenderis, M. Taylor and B.C. van Rees, Topologically massive gravity and the AdS/CFT correspondence, JHEP 09 (2009) 045 [arXiv: 0906.4926] [SPIRES].

[8] D. Grumiller and I. Sachs, $A d S_{3} / L C F T_{2}$ - correlators in cosmological topologically massive gravity, JHEP 03 (2010) 012 [arXiv: 0910.5241] [SPIRES].

[9] D. Grumiller and O. Hohm, $A d S_{3} / L C F T_{2}$ - correlators in new massive gravity, Phys. Lett. B 686 (2010) 264 [arXiv:0911.4274] [SPIRES].

[10] M. Alishahiha and A. Naseh, Holographic renormalization of new massive gravity, Phys. Rev. D 82 (2010) 104043 [arXiv: 1005.1544] [SPIRES].

[11] H. Lü and C.N. Pope, Critical gravity in four dimensions, Phys. Rev. Lett. 106 (2011) 181302 [arXiv:1101.1971] [SPIRES].

[12] S. Deser et al., Critical points of D-dimensional extended gravities, Phys. Rev. D 83 (2011) 061502 [arXiv:1101.4009] [SPIRES].

[13] M. Alishahiha and R. Fareghbal, D-dimensional log gravity, Phys. Rev. D 83 (2011) 084052 [arXiv:1101.5891] [SPIRES].

[14] E.A. Bergshoeff, O. Hohm, J. Rosseel and P.K. Townsend, Modes of log gravity, Phys. Rev. D 83 (2011) 104038 [arXiv: 1102 .4091] [SPIRES].

[15] M. Porrati and M.M. Roberts, Ghosts of critical gravity, Phys. Rev. D 84 (2011) 024013 [arXiv: 1104.0674] [SPIRES].

[16] H. Lü, Y. Pang and C.N. Pope, Conformal gravity and extensions of critical gravity, arXiv: 1106.4657 [SPIRES].

[17] S. Deser, R. Jackiw and S. Templeton, Topologically massive gauge theories, Ann. Phys. 140 (1982) 372 [Erratum ibid. 185 (1988) 406] [SPIRES]. 
[18] E.A. Bergshoeff, O. Hohm and P.K. Townsend, Massive gravity in three dimensions, Phys. Rev. Lett. 102 (2009) 201301 [arXiv:0901.1766] [SPIRES].

[19] V. Gurarie, Logarithmic operators in conformal field theory, Nucl. Phys. B 410 (1993) 535 [hep-th/9303160] [SPIRES].

[20] M. Flohr, Bits and pieces in logarithmic conformal field theory, Int. J. Mod. Phys. A 18 (2003) 4497 [hep-th/0111228] [SPIRES].

[21] M.R. Gaberdiel, An algebraic approach to logarithmic conformal field theory, Int. J. Mod. Phys. A 18 (2003) 4593 [hep-th/0111260] [SPIRES].

[22] A.M. Ghezelbash, M. Khorrami and A. Aghamohammadi, Logarithmic conformal field theories and AdS correspondence, Int. J. Mod. Phys. A 14 (1999) 2581 [hep-th/9807034] [SPIRES].

[23] I.I. Kogan, Singletons and logarithmic CFT in AdS/CFT correspondence, Phys. Lett. B 458 (1999) 66 [hep-th/9903162] [SPIRES].

[24] Y.S. Myung and H.W. Lee, Gauge bosons and the $A d S_{3} / L C F T_{2}$ correspondence, JHEP 10 (1999) 009 [hep-th/9904056] [SPIRES].

[25] P. Hořava, Membranes at quantum criticality, JHEP 03 (2009) 020 [arXiv: 0812.4287] [SPIRES].

[26] P. Hořava and C.M. Melby-Thompson, Anisotropic conformal infinity, Gen. Rel. Grav. 43 (2011) 1391 [arXiv:0909. 3841] [SPIRES].

[27] A. Hosseiny and S. Rouhani, Logarithmic correlators in non-relativistic conformal field theory, arXiv: 1001.1036 [SPIRES].

[28] A. Hosseiny and A. Naseh, On holographic realization of logarithmic GCA, arXiv:1101.2126 [SPIRES].

[29] M. Bianchi, D.Z. Freedman and K. Skenderis, Holographic renormalization, Nucl. Phys. B 631 (2002) 159 [hep-th/0112119] [SPIRES].

[30] K. Skenderis, Lecture notes on holographic renormalization, Class. Quant. Grav. 19 (2002) 5849 [hep-th/0209067] [SPIRES].

[31] M. Taylor, Non-relativistic holography, arXiv:0812.0530 [SPIRES].

[32] B.C. van Rees, Holographic renormalization for irrelevant operators and multi-trace counterterms, JHEP 08 (2011) 093 [arXiv: 1102.2239] [SPIRES].

[33] M. Guica, K. Skenderis, M. Taylor and B.C. van Rees, Holography for Schrödinger backgrounds, JHEP 02 (2011) 056 [arXiv:1008.1991] [SPIRES]. 